\title{
Density-Dependent Individual and Population-Level Metabolic Rates in a Suite of Single-Celled Eukaryotes
}

\author{
John P. DeLong* and David T. Hanson
}

Department of Biology, University of New Mexico, Albuquerque, New Mexico, 87131, USA

\begin{abstract}
Population level metabolic rates are by definition the sum of the individual metabolic rates within a population. Several studies have used estimates of individual metabolic rates to scale up metabolic activity of individuals to populations or whole communities. However, for aquatic single-celled organisms, individual metabolic rate is related to percapita resource availability, and accounting for this fact is essential for obtaining accurate estimates of population- or community-level metabolism. We frame the problem with a simple model of resource division that predicts per capita metabolic rate should decline with increasing density. We allow the magnitude of density-dependence to be adjusted by intraspecific competition, from perfectly dependent to completely independent of density. Our results demonstrate that per-capita metabolic rate of single-celled eukaryotes is indeed inversely related to density via the per-capita availability of resources, and this has a significant effect on population-level metabolic rates. Suppression of individual metabolic rate occurred up to an order of magnitude, and although this magnitude of suppression has been seen in starved protists, our results indicate that a broad continuum of density-dependence governs the resource-dependent variability in metabolic rates for these organisms. The species we used cover a range of resource acquisition modes and phylogenies, suggesting that density-dependence of metabolic rate may be widespread in aquatic unicells.
\end{abstract}

Keywords: Metabolic rate, protist, density-dependence, resource effects, population metabolism, Tetrahymena, Chlamydomonas.

\section{INTRODUCTION}

Population-level metabolism is the sum of the metabolic rates of all the individuals in a population. This fact has enabled researchers to generate estimates of community-level metabolic rate by summing up the metabolic rates of all the individuals in a population, and then summing across the populations in the community [1-4]. Recent efforts to "scale up" from the metabolism of individuals to communities have begun by estimating individual metabolic rate, $B$, from mass and temperature:

$$
B=b_{o} M^{\Theta} e^{\frac{E}{k_{b} T}}
$$

where $b_{\mathrm{o}}$ is a taxon-specific constant, $M$ is the average body mass of the organism $(\mathrm{g}), \Theta$ is an allometric scaling exponent, $E$ is the activation energy $(\mathrm{eV}), T$ is temperature (Kelvins), and $k_{b}$ is the Boltzmann constant (eV per Kelvin) [5]. Then, given an estimate of the number of individuals of each body size, one can integrate across all the organisms in the population or community and estimate the total metabolic rate.

For aquatic microorganisms, the metabolic rate of individuals is also related to environmental conditions, and in particular, to resource availability [6], up to a saturation point. Two recent studies estimated the metabolism of

*Address correspondence to this author at the Department of Biology, University of New Mexico, Albuquerque, New Mexico, 87131, USA; Tel: 505277-3431; Fax: 505-277-0304; E-mail: jdelong@unm.edu aquatic communities by adding photosynthetically active radiation (PAR) as a resource term $(R)$ to equation 1 :

$B=b_{o} M^{\Theta} e^{\frac{E}{k_{b} T}} R$

The first of these studies added PAR as a MichaelisMenten, saturating light response to predict community photosynthesis [7], and the second added PAR modified by a fitted exponent to predict community production [8]. Both of these approaches should produce improved estimates of community metabolism or production, relative to the original model without a resource term. The resource modification of equation 2 should be equally applicable to heterotrophic species for which metabolic rate may vary with food resources [9].

However, the metabolic rate of individuals may be more closely related to the per-capita availability of resources than to the total resources available to the population. This is because what a single organism experiences is its share of the available resource, not the entirety of the resource in the community. Equation 1 is designed to estimate the metabolic rate of an average individual, and it could be modified by adding a resource term that describes the change in average individual metabolic rate with per-capita resource availability. This modification requires a knowledge of how individual metabolic rates relate to per-capita resource availability, yet no study has assessed this relationship or attempted to model it. Because of this, we introduce a simple model that strives to logically relate per-capita resource availability to average per-capita metabolic rate. 
Resources are rarely constant in nature, but we begin with the simplification that they are. Consider that a population with a fixed amount of limiting resource, $R_{\text {tot }}$, available at any given time, divides those resources among all individuals in the population, $N$, such that the average per capita resource availability, $\bar{R}_{\mathrm{pc}}$, is inversely related to $N$. Given that these resources support metabolism, per capita metabolic rate, $\bar{B}_{\mathrm{pc}}$, should be proportional to $\bar{R}_{\mathrm{pc}}$, such that

$$
\bar{B}_{p c} \propto \bar{R}_{p c}=\frac{R_{t o t}}{N}
$$

this simple model predicts that across a wide range of $N$, from a single individual to carrying capacity, a broad negative relationship between $\bar{B}$ nc and $N$ should be observed, up to some maximum for both $\bar{R}_{\mathrm{pc}}$ and $\bar{B}_{\mathrm{pc}}$, which is perhaps set by a saturating ability to ingest food (e.g., a type 2 functional response). Thus, when resources are unlimited and densities are low, organisms may operate at a physiological $\bar{B}_{\max }$, but when densities rise and resources become more restricted, they should operate in the density-dependent manner described by equation 3 . It is possible that $\bar{B}$ pc is not proportional to $\bar{R}_{\mathrm{pc}}$, and is rather related to it in some nonlinear way. A modification of the model could be made to accommodate this possibility if a simple linear model inadequately describes experimental data.

Below $\bar{B}_{\max }$, equation 3 suggests that the addition of one individual to the population has the same effect whether it is added at low or at high density, which would be the case if body size, resource use, or energy allocation to alternative uses does not vary with density. If these things do change with density, however, it could be expected that deviation from a perfect inverse relationship would occur. This could be captured by introducing an intraspecific competition exponent, $\alpha_{11}$, on $N$. Our notation here is modeled after LotkaVolterra competition coefficients and can be read as the competitive effect of species one on itself (this notation is useful because, in principle, it could be extended to look at the effect of another species on the focal species):

$$
\bar{B}_{p c}=\frac{c R_{t o t}}{N^{\alpha_{11}}}
$$

where $c$ is a constant accounting for the conversion of resources to metabolism (metabolic rate gained per unit of resource). Because in practice it will be difficult to separate the parameters $R_{\text {tot }}$ and $c$, we use a combined parameter $C_{R}$ in this initial analysis. When intraspecific competition is perfect, $\alpha_{11}=1$, but if mechanisms prevent suppression of metabolic rate despite increases in density, $\alpha_{11}$ would decrease $\left(\alpha_{11}<1\right)$. At the extreme, metabolic rates could be completely independent of density $\left(\alpha_{11}=0\right)$. It is likely that most species would show $\alpha_{11}$ values somewhere between these two extremes, showing density-dependent metabolic rates along with mechanisms for maintaining metabolic rate in the face of intraspecific competition (Fig. 1A). However, it is also conceivable that $\alpha_{11}$ could be $<0$ if there is intraspecific facilitation or $>1$ if there are mechanisms that diminish access to resources beyond a simple division of resources mechanism. Nonetheless, we offer $0<\alpha_{11}<1$ as a useful starting point for our analyses.
The degree of dependence of per-capita metabolic rate on density has significant ramifications for population, and by extension, community metabolism. Population metabolism, $B_{\text {pop }}$, is defined as the product of the per-capita metabolic rate and the number of individuals. As such, estimates of $B_{\text {pop }}$ depend on how $\bar{B}_{\text {pc }}$ is related to $N$. Given the possibilities outlined above, population-level metabolism can fall between $\bar{B}_{\mathrm{pc}}$ invariance (metabolic rate independent of density, $\left.\alpha_{11}=0\right)$ and $B_{\text {pop }}$ invariance (metabolic rate proportional to the inverse of density, $\alpha_{11}=1$ ) (Fig. 1B). $\bar{B}_{\mathrm{pc}}$ invariance is the implicit assumption in all studies we have found that attempt to scale metabolism from individuals up to higher levels of ecological organization [1-4], including studies that considered resource availability as a predictor [7-8]. But if metabolic rates are density-dependent $\left(\alpha_{11}>0\right)$, then incorporating density-dependence is necessary to accurately scale metabolism up from individuals to higher levels of organization.

Here we test predictions generated by equation 4 with a suite of protists grown in microcosms. By holding body size and resource levels constant and manipulating density, we were able to evaluate the effect of density on metabolic rate and test the model. Due to difficulties measuring metabolic rates at low densities within a population context, we restrict our analyses to populations above very low $N$, and therefore below the maximum metabolic rates. Specifically, we examine 1) whether $\bar{B}_{\mathrm{pc}}$ is inversely related to $N, 2$ ) what values of intraspecific competition are shown $\left(\alpha_{11}\right)$, and 3$)$ whether $B_{\text {pop }}$ shows indications of $B_{\text {pop }}$ invariance or $\bar{B}$ pc invariance. Using new experimental data augmented by data from published studies, we found strong support for the model. Metabolic rates were density dependent $\left(\alpha_{11}>0\right)$ at both the percapita and population level, suggesting that incorporating these effects into population and community metabolism studies is warranted.

\section{MATERIALS AND METHODS}

\section{Culturing}

We investigated the effect of population density on metabolic rate using four species of protist: Chlamydomonas reinhardtii, Galdieria sulphuraria, Tetrahymena pyriformis, and Blepharisma sp. Each species was batch-cultured using standard species-specific medium and allowed to grow to a late-growth phase prior to measurements. Details for each species are as follows:

C. reinhardtii was grown in axenic cultures in TAP (TrisAcetate Phosphate) medium [10]. Populations were grown in $\sim 25 \mathrm{~mL}$ of medium in 200-mL flasks kept stirred at room temperature $\left(\sim 20^{\circ} \mathrm{C}\right)$, with light exposure of $15 \mu \mathrm{mol}$ photons $\mathrm{m}^{-2} \mathrm{~s}^{-1}$ and with a $24-\mathrm{hr}$ photoperiod. Flasks were capped with sponge filters topped with aluminum foil to maintain sterility and gas exchange. Populations were grown in the light; sample vials were wrapped in aluminum foil to maintain dark conditions for measuring respiration. A population was initiated with a small aliquot from a stationaryphase culture and was allowed to grow for 11 days before sampling. No replacement or addition of new medium was made during the culturing period. G. sulphuraria was grown and sampled in the same manner as $C$. reinhardtii, except 

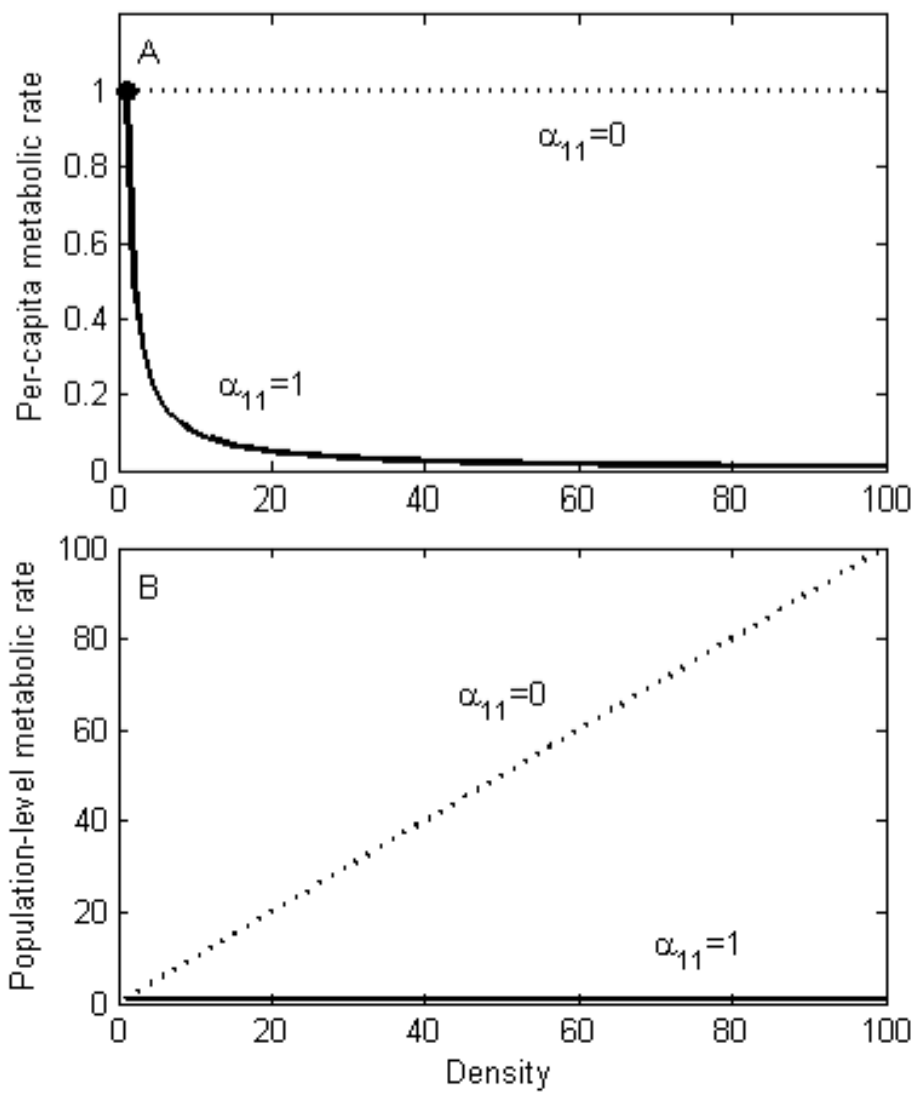

Fig. (1). Theoretical extremes of the relationship between density and metabolic rate. A) $\bar{B}_{\mathrm{pc}}$ that is independent of density (dotted line) is fixed at the maximum rate (shown here as 1 , black dot). Density-dependent $\bar{B}_{\mathrm{pc}}$ (solid line) shows the inverse dependence of metabolic rate as predicted by equation 4 . B) $B_{\text {pop }}$ that results from density-dependent metabolic rate ( $B_{\text {pop }}$ invariance, solid line) and from densityindependent per-capita rates ( $\bar{B}_{\mathrm{pc}}$ invariance, dotted line).

that the growth medium was modified SAG [11] and the population was allowed to grow $\sim 30$ days before sampling.

T. pyriformis was grown axenically in Tetrahymena medium (Carolina Biological Supply). The 10-mL culture was grown in a 16-mL vial sealed with a septum top and bubbled with filtered $(0.2 \mu \mathrm{m}$ syringe filter $)$ air to maintain sterile, aerobic conditions. The temperature of the microcosm was maintained at $20^{\circ} \mathrm{C}$ in a water bath. To reduce the potential for contamination, we added penicillin (10,000 I.U.) and streptomycin $\left(10,000 \mu \mathrm{g} \mathrm{mL}^{-1}\right)$ at a $1: 100$ dilution with the food medium. The population was allowed to grow for $\sim 14$ days prior to sampling.

Blepharisma sp. was obtained in a monoxenic culture from Carolina Biological Supply. This population was grown for $\sim 10$ days at $\sim 20^{\circ} \mathrm{C}$ after receipt of the cultures. Blepharisma was cultured with its bacterial food source. Samples were placed in a filter apparatus and were washed with sterile protozoan medium (Carolina Biological Supply) prior to measurement. The large Blepharisma ( 200 $\mu \mathrm{m}$ in length) were retained in the filter while the bacteria and other small contaminants were washed out with the filtrate. Thus, the samples were not completely sterile, but the impact of bacteria on measurements of oxygen consumption was minimized.

\section{Sampling}

We began by taking $\sim 1.5 \mathrm{~mL}$ samples, drawn without replacement, from the batch cultures at our starting-point density (late growth phase). We altered the cell density of these samples by gentle $(1-2,000 \mathrm{rpm})$ centrifugation. After centrifuging, we took a $0.7 \mathrm{~mL}$ sub-sample of either the supernatant (reduced density) or of the concentrated portion (augmented density) for measurement. Centrifuge times were altered to increase (concentrate) or decrease (supernatant) densities. Samples were density-manipulated and set up for oxygen measurements within a few minutes. All replicates were measured over an approximately 1-day period, to isolate the effect of density and preclude long-term changes in cell size, resource concentration, or physiological state of cells in the culture.

\section{Density}

The approach for measuring density varied across species because of body size, cell motility, and population density. For $C$. reinhardtii and $G$. sulphuraria, densities were determined by repeated sample counts in a hemocytometer. For $T$. pyriformis and Blepharisma, individuals were counted under a compound microscope. For T. pyriformis, two $0.1-\mathrm{mL}$ sub samples were extracted from the samples in which oxygen consumption was measured. Volumes extracted were measured with an electronic balance, and if necessary dilutions with the resource medium were made until cell densities were low enough to count. For Blepharisma, dilutions were not necessary, and the entire oxygen consumption sample was counted. 


\section{Metabolic Rate}

We used a fluorescent oxygen probe (FOXY-18G, Ocean Optics Inc., Dunedin, Florida, USA) to measure the change in molar fraction of dissolved oxygen in culture samples due to metabolic activity. The probe does not consume oxygen and thus can be used to directly observe changes in oxygen levels in the samples. We calibrated the system using $\mathrm{N}_{2}$ gas and $20.9 \% \mathrm{O}_{2}$ air. We measured the change in oxygen in a $\sim 0.65 \mathrm{~mL}$ well-mixed sample sealed with no headspace and maintained at $20^{\circ} \mathrm{C}$ in a stabilized water bath. We converted the molar fraction of oxygen measured at each time step to a mole concentration of oxygen with,

$$
\text { moles } \mathrm{O}_{2}=\frac{\mathrm{O}_{2} \text { molar fraction } * \text { moles } \mathrm{N}_{2}}{1-\mathrm{O}_{2} \text { molar fraction }}
$$

We assumed an equilibrium value for the moles of $\mathrm{N}_{2}$ dissolved in the sample given the average atmospheric pres- sure in Albuquerque ( $80 \mathrm{kPa})$, New Mexico, and $20^{\circ} \mathrm{C}$. The rate of oxygen consumption, $B_{\text {pop }}$, was then calculated using linear regression of mole quantity on time. Initial molar concentration of oxygen was $13-25 \%$, with reductions of $0.5-3 \%$ over the course of the experiments, which lasted less than one hour, and the oxygen consumption curves did not change during the measurement period. Per capita metabolic rate, $\bar{B}_{\mathrm{pc}}$, was calculated by dividing the whole-population oxygen consumption rate by density.

\section{Additional Data from the Literature}

The metabolic rate of $T$. pyriformis also was measured at $30^{\circ} \mathrm{C}$ by Finlay et al. [12] during a population growth cycle using a Warburg apparatus (Fig. (2) in [12]). The original metabolic rate units were $\mathrm{nL} \mathrm{O}_{2}$ consumed $\mathrm{h}^{-1}$. We captured the data from the figure with digital imaging software. The data were presented originally as time-series of density and oxygen consumption. The shared time points were used to
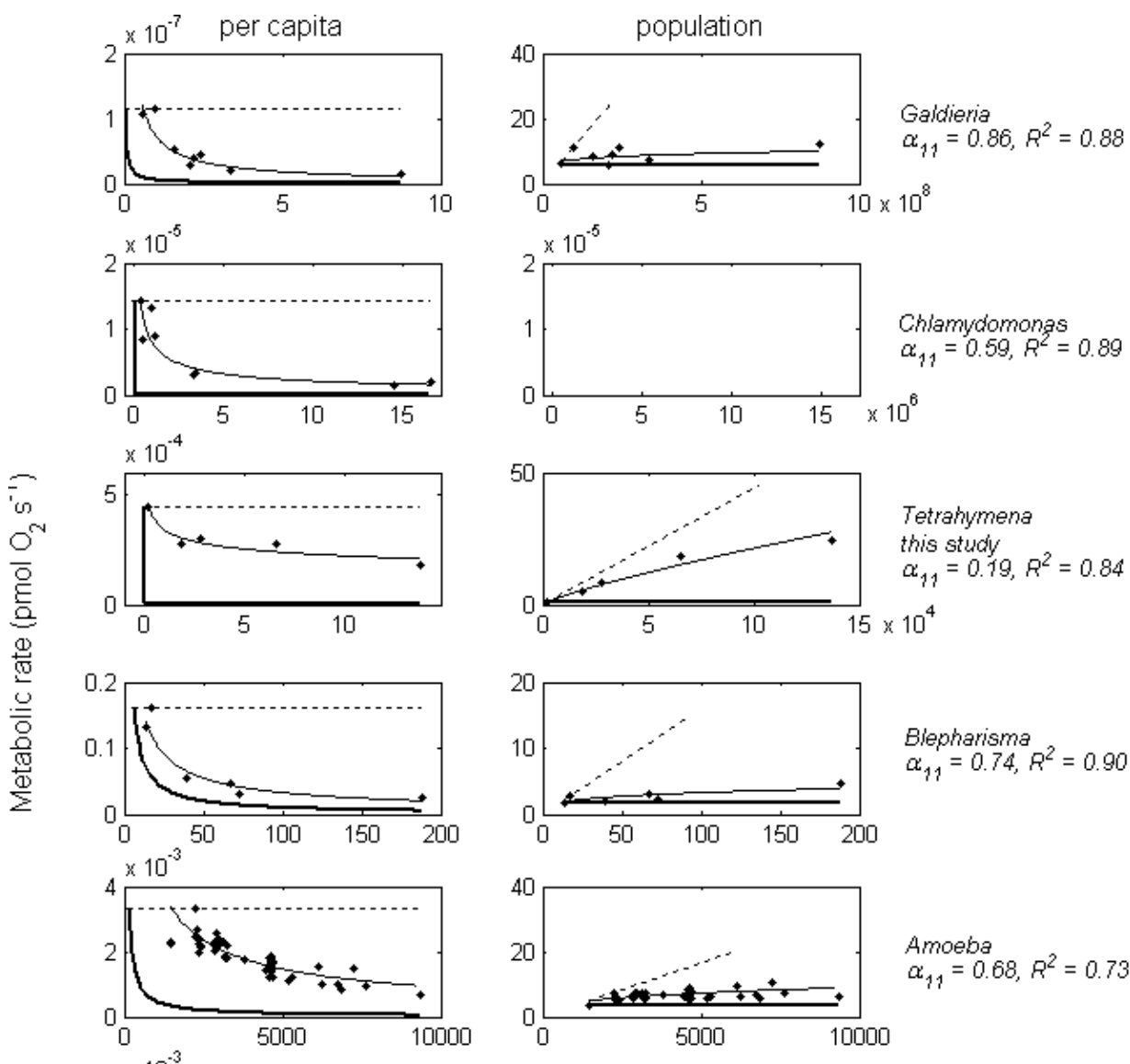

Blepharisma

$\alpha_{11}=0.74, R^{2}=0.90$
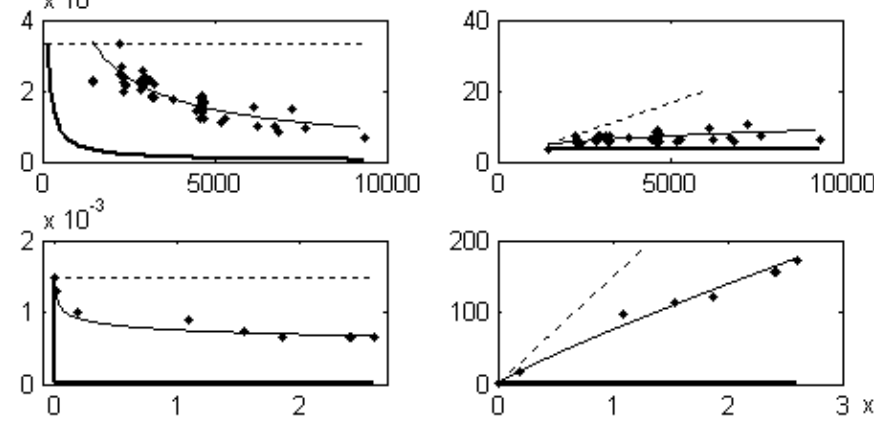

Amoeba

$\alpha_{11}=0.68, R^{2}=0.73$

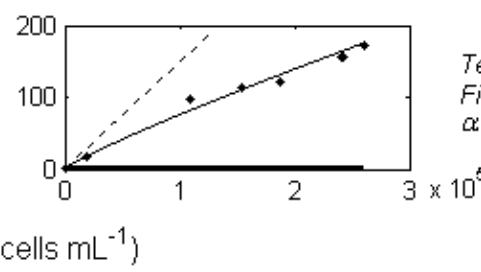

Tetrahymena

Finlay et al. 1983

$\alpha_{11}=0.12, R^{2}=0.94$

Density (cells $\mathrm{mL}^{-1}$ )

Fig. (2). Per capita metabolic rates ( $\bar{B}_{\text {pc }}$; left column) and population-level metabolic rates ( $B_{\text {pop }}$; right column) are density-dependent in single-celled eukaryotes. Panels show five species of protists: two autotrophs (G. sulphuraria and $C$. reinhardtii) and three heterotrophs (T. pyriformis, Blepharisma sp., and A. proteus). Thin lines represent fits of equation 4 ; dashed lines represent density-independent $\bar{B}_{\mathrm{pc}}\left(\alpha_{11}=0\right)$ for highest measured value; and thick line represents complete inverse dependence of $\bar{B}_{\mathrm{pc}}$ on density $\left(\alpha_{11}=0\right)$. Values of $\alpha_{11}$ are shown as well as $R^{2}$ values calculated for the per-capita plots. Because all species showed density-dependent $\bar{B}_{\text {pc }}$, population rates are all lower than would be expected with a density-independent estimate of $\bar{B}_{\mathrm{pc}}$. 
connect the density and the oxygen consumption data: if both an oxygen-consumption and a density measurement were reported for the same time, the density and metabolic rate data were paired.

Data for density and metabolic rates of A. proteus came from Nässberger and Monti [13]. The data presented in their Fig. (4) were digitized with digital imaging software. The metabolic rate was measured with a microcalorimeter at $25^{\circ} \mathrm{C}$, with densities adjusted by manually adding cells to the measurement ampoule. The number of cells in the ampoule was given in the figure, but the volume was not given, so for this species we simply assume the number of cells is a proportional index of density, assuming constant volume of their sample ampoules. The original metabolic rates were $\mathrm{nW}$ of heat produced, and these were converted to match the units we report for oxygen consumption (pmol $\mathrm{O}^{2} \mathrm{~s}^{-1}$ ), using equivalents of $1 \mathrm{cal}=4.184 \mathrm{~J}$ and $1 \mathrm{~mL} \mathrm{O}_{2}$ consumption yields 4.825 cal.

\section{RESULTS}

For all species, $\bar{B}_{\mathrm{pc}}$ was inversely related to $N$, as predicted by the resource-division model (equation 4 , Fig. 2). Density had a large negative effect on $\bar{B}_{\mathrm{pc}} \bar{B}_{\mathrm{pc}}$ ranged up to an order of magnitude in response to density manipulations for Galdieria, Chlamydomonas, and Blepharisma, while Tetrahymena (both studies) and Amoeba showed 2-3-fold variation. All experiments, including our new data and data from the literature, showed that $\bar{B}_{\mathrm{pc}}$ was an inverse function of $N$. Fits of the power function from equation 4 were exceptionally tight, with $R^{2}$ ranging $0.73-0.94$, indicating that the model correctly captures the behavior of these systems.

There was considerable variation in $\alpha_{11}$, from 0.12 to 0.86 , with Tetrahymena showing the smallest values of $\alpha_{11}$ (most $\bar{B}_{\mathrm{pc}}$ invariant) and Galdieria showing the largest values of $\alpha_{11}$ (most $B_{\text {pop }}$ invariant). All $\bar{B}_{\text {pc }}$ data fell between the two extremes of per-capita metabolic invariance and population-level metabolic invariance (Fig. 2, left column). Similarly, $B_{\text {pop }}$ values fell between either extreme, where population-level metabolic rates increased neither linearly with density nor showed perfect invariance (Fig. 2, right column).

The oxygen probe we used produced rapid ( 10 $\mathrm{min})$ and linear trends in oxygen consumption, and was capable of detecting subtle changes in rate. A test of the probe with a sample of Chlamydomonas over a range of light conditions showed oxygen consumption rates that were distinct, linear, and differentiable over small time frames. The other approaches to measuring metabolic rate gave qualitatively the same patterns as produced with our methods. Nässberger and Monti's [13] study with Amoeba showed the same densitydependent pattern of $\bar{B}_{\mathrm{pc}}$ using direct microcalorimetry of heat production, and Finlay et al.'s [12] study on Tetrahymena using a Warburg apparatus produced a pattern of density-dependent $\bar{B}_{\mathrm{pc}}$ that was quantitatively similar to our experiment with Tetrahymena, even showing similar values for $\alpha_{11}\left(\alpha_{11}=0.19\right.$ from our data and $\alpha_{11}=0.12$ from Finlay et al.'s [12] data).

The three methods of altering density in these studies also produced similar patterns. The study with Amoeba [13] and Finlay et al.'s [12] Tetrahymena study did not use cen- trifuges to alter density. Amoeba was concentrated by manually adding cells, and Tetrahymena was allowed to grow through time, yet these studies showed the same patterns as our experiments with manipulated densities. In addition, we do not think that gently centrifuging cells altered the resources available to cells, either through cell lysis, which we did not observe, or through concentrating nutrients. If centrifuging reduced nutrient availability to the non-concentrated cells, then the low-density cultures would have had low resource availability, and vice versa, which would create patterns opposite of those we observed. Thus, the patterns we report here are robust to metabolic rate measurement and density-manipulation technique.

\section{DISCUSSION}

We introduced a simple model built upon the idea that a limiting resource must be divided up among the individuals in a population. Because of this, and below a maximum resource consumption level and metabolic rate, individuals will become more resource-limited as density increases. If resources are metabolism supporting, then $\bar{B}_{\mathrm{pc}}$ should be proportional to per-capita resource availability and should follow the same inverse function of density as per-capita resource availability. Our model includes an intraspecific competition exponent, $\alpha_{11}$, which allows us to quantify the per-capita effect of adding individuals to the population. For all species investigated here, including both our new experimental data and data from the literature, increased population density resulted in reduced $\bar{B}_{\mathrm{pc}}$, as predicted by the model. However, we advocate for further testing of the model, by altering resource levels while holding density constant, or by altering resources and density simultaneously, and measuring the subsequent effect on metabolic rates.

Our results are the first to illustrate a broad, general picture of the relationship between density and metabolic rate in protists. The literature is replete with studies on the metabolic rates of protists, and some have observed that the density of cells in a sample negatively affects the rate [13-17]. McCashland and Kronschnabel [17] suggested that competition for nutrients was the cause, foreshadowing our results here. But mostly, the observation has usually taken the form of a technical comment, where the recognition of a negative relationship led to the use of certain densities to get "good" rates. In contrast, Reich [18] found no effect of density on metabolic rate in the amoeba Mayorella palestinensis and attributed previous findings to technical errors. It is likely that Reich missed the effect because a wide range of densities was not tested. Our results reinforce previous observations, but more importantly, they show that there is a broad pattern that can be captured by a simple model of resource division. The model provides a mechanism to quantify the density-dependence (value of $\alpha_{11}$ ), and allows us to ask how it may vary. For example, we think it would be interesting to determine if $\alpha_{11}$ varies with temperature or other environmental conditions.

The density-dependence of metabolic rate is likely to pertain to a wide range of microorganisms. The species we used were both heterotrophs (Blepharisma, Amoeba, and Tetrahymena) and autotrophs (Galdieria and Chlamydomonas), and because they are phylogenetically broad, including red algae (Galdieria), green algae (Chlamydomonas), 
ciliates (Blepharisma and Tetrahymena), and rhizopods (Amoeba), we think that density-dependent suppression of metabolic rate is likely to be widespread in aquatic unicells whenever resource constraints apply. Furthermore, we do not think that the phenomenon of density-dependent metabolic rate need be restricted to single-celled organisms. The individual metabolic rates of many types of organisms, including vertebrates, can vary enormously. Food, nutrient, and light availability vary for most organisms, and some variability in metabolic rate may result. For example, density appears to have a strong effect on the metabolic rate of the daphnid Simocephalus vetulus [19], but more evaluation is needed with other taxa.

One consequence of the density-dependence of metabolic rate is that population-level metabolic rate depends critically on how per capita metabolic rate relate to density. Population-level metabolic rates for all populations studied here were intermediate between $\bar{B}_{\mathrm{pc}}$ and $\bar{B}_{\text {pop }}$ invariance (Fig. 2), and all population-level rates were lower than what would have been predicted using the assumption of $\bar{B}_{\mathrm{pc}}$ invariance, which is a standard assumption for scaling metabolism up to populations and communities. When such scalingup approaches are used, some understanding of the dependence of $\bar{B}_{\mathrm{pc}}$ on density is necessary to obtain accurate scaled-up estimates. Approaches that add resource terms to equation 1 to improve individual metabolic rate estimates may be more fruitful if they employ a density-dependent mechanism like the one we suggest here (equation 4).

For many species in natural settings, the assumption of fixed resource supply rates is not valid. Resource availability in aquatic communities varies through time as a result of diurnal patterns, hydrology, and a wide range of other factors. In our laboratory experiments, a constant resource assumption was reasonable, and our manipulation of density allowed us to ignore $R_{\mathrm{tot}}$ and test a specific prediction of the model. However, the model also predicts that, holding density constant, increasing $R_{\mathrm{tot}}$ should linearly increase percapita metabolic rate. This effect has been observed in laboratory cultures of Astasia longa [20], but further investigation of resource effects is necessary. Ultimately, we think the model we present, and the finding of density-dependent metabolic rates, should influence the way we think about and model population- and community-level metabolism, at least in communities of aquatic microorganisms.

\section{ACKNOWLEDGEMENTS}

We thank L. Green, U. Bergthorrsen, and P. Morin for technical assistance. J. Brown, O. Burger, T. Meehan, B. Milne, and several anonymous reviewers gave helpful comments on this work. JPD received funding from the UNM, Department of Biology, Summer Grove Scholarship and
GRAC and SRAC graduate student panels. Funding was also provided by UNM RAC grant \#05-L-07 and NMTRC funding to DTH.

\section{REFERENCES}

[1] Finlay BJ. Community production and respiration by ciliated protozoa in the benthos of a small eutrophic loch. Fresh Biol 1978; 8: 327-41.

[2] Enquist BJ, Economo EP, Huxman TE, Allen AP, Ignace DD, Gillooly JF. Scaling metabolism from organisms to ecosystems. Nature 2003; 423: 639-42.

[3] Ernest SKM, Enquist BJ, Brown JH, et al. Thermodynamic and metabolic effects on the scaling of production and population energy use. Ecol Lett 2003; 6: 990-95.

[4] DeLong JP. The maximum power principle predicts the outcomes of two-species competition experiments. Oikos 2008; 117: 132936.

[5] Gillooly JF, Brown JH, West GB, Savage VM, Charnov EL. Effects of size and temperature on metabolic rate. Science 2001; 293 : 2248-51.

[6] Finlay B. In: del Giorgio PA, William, PJ le B, Eds, Respiration in aquatic protists. Respiration in aquatic ecosystems. Oxford: UK 2005; pp. 47-56.

[7] López-Urrutia A, San Martin E, Harris RP, Irigoien X. Scaling the metabolic balance of the oceans. Proc Natl Acad Sci USA 2006 103: 8739-44.

[8] de Castro F, Gaedke U. The metabolism of lake plankton does not support the metabolic theory of ecology. Oikos 2008; 117: 121826.

[9] Fenchel T, Finlay BJ. Respiration rates in heterotrophic, free-living protozoa. Microb Ecol 1983; 9: 99-122.

[10] Harris E. The Chlamydomonas sourcebook. San Diego, USA 1989.

[11] Allen MB. Studies with Cyanidium caldarium, an anomalously pigmented chlorophyte. Arch Microb 1959; 32: 270-77.

[12] Finlay B, Span A, Ochsenbein-Gattlen C. Influence of physiological state on indices of respiration rate in protozoa. Comp Biochem Phys 1983; 74: 211-9.

[13] Nässberger L, Monti M. Assessment of overall metabolism In Amoeba proteus measured by a microcalorimetric method. Protoplasma 1984; 123: 135-9.

[14] Specht H. Aerobic respiration in Spirostomum ambiguum and the production of ammonia. J Cell Comp Phys 1934; 5: 319-33.

[15] Pace DM, Kimura KK. The effect of temperature on respiration in Paramecium aurelia and Paramecium caudatum. J Cell Comp Phys 1944; 24: 173-83.

[16] Pace DM, Lyman ED. Oxygen consumption and carbon dioxide elimination in Tetrahymena geleii fergason. Biol Bull 1947; 92: 210-6.

[17] McCashland BW, Kronschnabel JM. Exogenous factors affecting respiration in Tetrahymena pyriformis. J Euk Microb 1962; 9: 2769.

[18] Reich K. Studies on the respiration of an amoeba, Mayorella palestinensis. Physiol Zool 1948; 21: 390-412.

[19] Hoshi T. Studies on physiology and ecology of plankton xii. changes in $\mathrm{O}_{2}$-consumption of the daphnid, Simocephalus vetulus, with the decrease of $\mathrm{O}_{2}$-concentration. Sci Rep Tokohu Univ, Fourth Series, Biol 1957; 23: 27-33.

[20] Wilson BW. The oxidative assimilation of acetate by Astasia longa and the regulation of cell respiration. J Cell Comp Phys 1963; 62: 49-56. 